\title{
Bradykinin promotes proliferation, migration, and invasion of cervical cancer cells through STAT3 signaling pathways
}

\author{
WEI WANG, YING ZHOU, RUI WEI, GUIYING JIANG, FEI LI, \\ XI CHEN, XUEQIAN WANG, DING MA and LING XI
}

\begin{abstract}
Department of Obstetrics and Gynecology, Tongji Hospital, Tongji Medical College, Huazhong University of Science and Technology, Wuhan, Hubei 430030, P.R. China
\end{abstract}

Received May 31, 2019; Accepted September 5, 2019

DOI: $10.3892 /$ or.2019.7380

\begin{abstract}
It has been reported recently that bradykinin (BK) is involved in the regulation of various processes in cancer cells. However, its role and underlying mechanism of action in cervical cancer (CC) are still unknown. In the present study, it was revealed that $\mathrm{BK}$ promoted proliferation, migration, and invasion of $\mathrm{CC}$ cells, whereas bradykinin $\mathrm{B} 2$ receptor antagonist HOE140 had the inverse effect. Furthermore, it was confirmed that overexpression of bradykinin $\mathrm{B} 2$ receptor (B2R) facilitated the proliferation, migration, and invasion of BK-treated CC cells, while knockdown of B2R had the opposite effect. Mechanistically, the present results revealed that the BK/B2R-induced biological function of $\mathrm{CC}$ cells occured by activating STAT3 signaling pathways, and that knockdown of B2R or B2R antagonist had the opposite effects. Moreover, it was demonstrated that BK/B2R facilitated CC cell migration and invasion by upregulating the expression of the STAT3-regulated products MMP2 and MMP9, while downregulating the expression of the pro-apoptotic protein cleaved caspase-9. Thus, the present findings revealed that BK promoted CC cell proliferation, migration, and invasion by binding to B2R via STAT3 signaling pathways.
\end{abstract}

\section{Introduction}

Cervical cancer (CC) is one of the most common malignancies worldwide. Although screening for $\mathrm{CC}$ is widespread, over 500,000 new cases of CC were estimated to occur worldwide in 2018, thus ranking it as the second most common malignant tumor in females (1). With advances in surgery, radiotherapy, and chemotherapy, the five-year survival rate of patients

Correspondence to: Professor Ling Xi, Department of Obstetrics and Gynecology, Tongji Hospital, Tongji Medical College, Huazhong University of Science and Technology, 1095 Jiefang Avenue, Wuhan, Hubei 430030, P.R. China

E-mail: 1xi@tjh.tjmu.edu.cn

Key words: bradykinin, bradykinin B2 receptor, STAT3, matrix metallopeptidase, cervical cancer receiving standardized treatment can reach $80 \%$. However, once patients develop metastases, the five-year survival rate drops below $50 \%$ (2). Thus, a deeper understanding of the mechanisms of CC development and identification of its critical signaling markers as potential targets for improved treatment strategies is crucial.

Bradykinin (BK) is an active peptide that is generated by the kallikrein-kinin system (KKS) (3). It has been reported that $\mathrm{BK}$ is involved in the regulation of various cellular processes in cancer cells, including cell proliferation and angiogenesis $(4,5)$. BK also facilitates cancer migration and invasion by stimulating the activity of membrane matrix metalloproteases (MMPs) and integrins $(6,7)$. The effect of $\mathrm{BK}$ is mediated via two $\mathrm{G}$ protein-coupled receptors, $\mathrm{B} 1$ and B2, which have been pharmacologically characterized and defined by molecular cloning (8). Furthermore, recent studies have indicated that activation of the bradykinin $\mathrm{B} 2$ receptor (B2R) by BK is involved in most BK-mediated biological actions (9-11). Studies performed by our group demonstrated that BK/B2R promoted angiogenesis of cervical cancer and facilitated tumorigenesis (12). In the present study, other underlying molecular mechanisms by which BK promotes tumor progression in $\mathrm{CC}$ were explored.

Signal transducer and activator of transcription 3 (STAT3) is persistently activated in many cancers and animal cancer models (13). Studies have also shown that STAT3 is constitutively activated in CC and cervical high-grade lesions $(14,15)$. STAT3 Tyrosine705 phosphorylation actives this canonical pathway, and results in malignant transformation by promoting cell proliferation, angiogenesis, invasion, and metastasis (16-18). The breakdown of the basement membrane and remodeling of the extracellular matrix (ECM) are markers for the invasion and metastasis of malignant tumors. The degradation of ECM proteins is mainly accomplished by a variety of MMPs $(19,20)$. Activated STAT3 upregulates MMP expression and activity $(15,21)$ and downregulates expression of pro-apoptotic proteins (22), thus promoting metastasis and proliferation in many cancers. Therefore, the expression of BK and B2R was detected, and their relationship with STAT3 was explored in $\mathrm{CC}$. The effect of BK treatment on CC cells was examined and the function of B2R was further characterized in BK-mediated effects. It was also demonstrated that BK promoted the proliferation, migration, and invasion of $\mathrm{CC}$ 
cells by activating STAT3 in vitro. These results highlighted the important role of $\mathrm{BK} / \mathrm{B} 2 \mathrm{R}$ in $\mathrm{CC}$.

\section{Materials and methods}

Cell lines and cultures. Human cervical cancer cell lines ( $\mathrm{SiHa}$ and $\mathrm{HeLa}$ ) were purchased from the American Type Culture Collection. The cells were maintained in Dulbecco's modified Eagle's medium (DMEM) supplemented with $10 \%$ fetal bovine serum (FBS). All the cells were cultured at $37^{\circ} \mathrm{C}$ in a humidified atmosphere containing $5 \% \mathrm{CO}_{2}$.

Transduction with lentiviruses. B2R-overexpressed and -knockdown cell lines were constructed as previously described (12). Target cells were selected with puromycin $(2 \mu \mathrm{g} / \mathrm{ml})$ for $48 \mathrm{~h}$. B2R expression was detected using reverse transcription-quantitative polymerase chain reaction (RT-qPCR) and western blotting. All lentiviruses were obtained from Shanghai GeneChem Co., Ltd. Cell experiments were finished within three months of cell construction.

RNA isolation and RT-qPCR. Total RNA was extracted from cells using a Total RNA Kit (Omega Bio-tek) and reverse transcribed into cDNA using M-MLV reverse transcriptase (Takara Bio, Inc.). Real-time quantitative PCR was performed using a Bio-Rad CFX96 (Bio-Rad Laboratories, Inc.) system with SYBR Green (Bio-Rad Laboratories, Inc.) in triplicate. Each sample was normalized to the control gene GAPDH. The primer sequences for PCR are presented as follows: human BDKRB2 forward, 5'-CCGAAAGAAGTCTTGGGAGGT-3' and reverse, 5'-CTGGCGTTCCACGGAGATG-3'; human GAPDH forward, 5'-GACAGTCAGCCGCATCTTCT-3' and reverse, 5'-TTAAAAGCAGCCCTGGTGAC-3'. The cycling conditions were as follows: $95^{\circ} \mathrm{C}$ for $3 \mathrm{~min}$, then 45 cycles of $95^{\circ} \mathrm{C}$ for $15 \mathrm{sec}$ and $60^{\circ} \mathrm{C}$ for $30 \mathrm{sec}$.

Western blot analysis. Cells were lysed in radioimmunoprecipitation assay (RIPA) lysis buffer (Beyotime Institute of Biotechnology) supplemented with a protease inhibitor cocktail (Roche Diagnostics). Concentrations of the proteins extracted from cell lines were determined using the Coomassie brilliant blue G-250 (BioFroxx; neoFroxx GmbH) staining method. Equal amounts of cell lysate $(30 \mu \mathrm{g})$ were resolved by $10 \%$ sodium dodecyl sulfate polyacrylamide gel electrophoresis (SDS-PAGE) and transferred to a polyvinylidene difluoride membrane. The blots were blocked with $5 \%$ bovine serum albumen (BSA) at room temperature for $1 \mathrm{~h}$ and then incubated with primary antibodies overnight at $4^{\circ} \mathrm{C}$. After washing three times, appropriate secondary antibodies were added, followed by incubation for $1 \mathrm{~h}$ at room temperature. Primary antibodies were as follows: p-STAT3 (dilution 1:2,000; product no. 9145T) and STAT3 (dilution 1:2,000; product no. 4904T; both from Cell Signaling Technology, Inc.), MMP2 (dilution 1:1,000; product code ab92536; Abcam), MMP9 (dilution 1:600; cat. no. 10375-2-AP; ProteinTech Group, Inc.), caspase-9 (dilution 1:1,000; product no. 9504T) and cleaved caspase-9 (dilution 1:1,000; product no. 9509T; both from Cell Signaling Technology, Inc.), BDKRB2 (dilution 1:500; product code ab134118; Abcam), GAPDH (dilution 1:2,000, cat. no. AC001; ABclonal Technology), and $\alpha$-tubulin (dilution 1:2,000; cat. no. A41641; Antgene). The proteins were detected using an enhanced chemiluminescence system (Pierce; Thermo Fisher Scientific, Inc.). The statistical data on protein levels in western blots were analyzed using ImageJ 1.8.0 software (National Institutes of Health).

Plate colony formation assay. Cells were digested and diluted with DMEM. For each group, 800 cells in $2 \mathrm{ml}$ DMEM were seeded in six-well culture plates and then maintained in a 5\% $\mathrm{CO}_{2}$ incubator at $37^{\circ} \mathrm{C}$. Various concentrations of BK $(1,2.5$ and $5 \mu \mathrm{M}$ ) and the bradykinin B2 receptor antagonist HOE140 (Tocris Bioscience) $(1,5$ and $10 \mu \mathrm{M})$ were added. Constructed cells were treated with $2.5 \mu \mathrm{M}$ BK. The culture plates were collected 12 days later, and the clones were stained with $0.1 \%$ crystal violet for $10 \mathrm{~min}$ at room temperature for visualization. Experiments were performed in triplicate.

Migration assays. Using a 24-well Transwell plate containing PET membranes with $8-\mu \mathrm{m}$ pores (Corning Inc.), CC cells were added into the upper chambers with non-coated membranes. Approximately $1 \times 10^{4}$ cells in $100 \mu 1$ of serum-free DMEM were placed in the upper chamber, and $500 \mu \mathrm{l}$ of the same medium containing BK ( $5 \mu \mathrm{M}$; Tocris Bioscience) was placed in the lower chamber. For cells transduced with lentiviruses, $500 \mu 1$ medium supplemented with $20 \% \mathrm{FBS}$ and $2.5 \mu \mathrm{M}$ BK was placed into the lower chambers. After $24 \mathrm{~h}$ at $37^{\circ} \mathrm{C}$, the cells that migrated into the underside of the membrane were fixed in $4 \%$ paraformaldehyde for $15 \mathrm{~min}$, stained with $0.5 \%$ crystal violet for $20 \mathrm{~min}$, and washed with PBS. At least six random fields were assessed under an Olympus IX73 microscope (Olympus Corp.) (x10, magnification). Experiments were performed in triplicate.

Invasion assay. The procedure for the invasion assay was similar to the migration assay described above, except that the upper chambers were coated with Matrigel $(10 \mathrm{mg} / \mathrm{ml}$; Corning Incorporated) diluted five times in serum-free DMEM. Briefly, the cells were placed in the upper chamber, and medium containing BK $(5 \mu \mathrm{M})$ or $20 \%$ FBS with $2.5 \mu \mathrm{M}$ BK was placed into the lower chambers. After $36 \mathrm{~h}$ at $37^{\circ} \mathrm{C}$, the cells were fixed, stained, washed, and counted. Experiments were performed in triplicate.

Scratch assay. Cells were added to six-well plates and then cultured in a $5 \% \mathrm{CO}_{2}$ incubator at $37^{\circ} \mathrm{C}$. Once the cell density reached $80 \%$, a vertical wound was produced in the monolayer and BK $(5 \mu \mathrm{M})$ or HOE140 $(10 \mu \mathrm{M})$ were added. Constructed cells were treated with $2.5 \mu \mathrm{M}$ BK. The closure of the wound was monitored at $0,24,36,48$, and $60 \mathrm{~h}$ and measured using ImageJ 1.8.0 software (National Institutes of Health). For each experiment, at least three scratched fields were recorded, and all scratch assays were performed in triplicate (x10, magnification).

Statistical analysis. The data are reported as the means \pm standard error. Statistical evaluation between groups was conducted with the Student's t-test. Comparisons of more than two groups were performed using one-way analysis of variance (ANOVA) followed by Tukey's post hoc test. Differences were considered statistically significant at $\mathrm{P}<0.05$. 

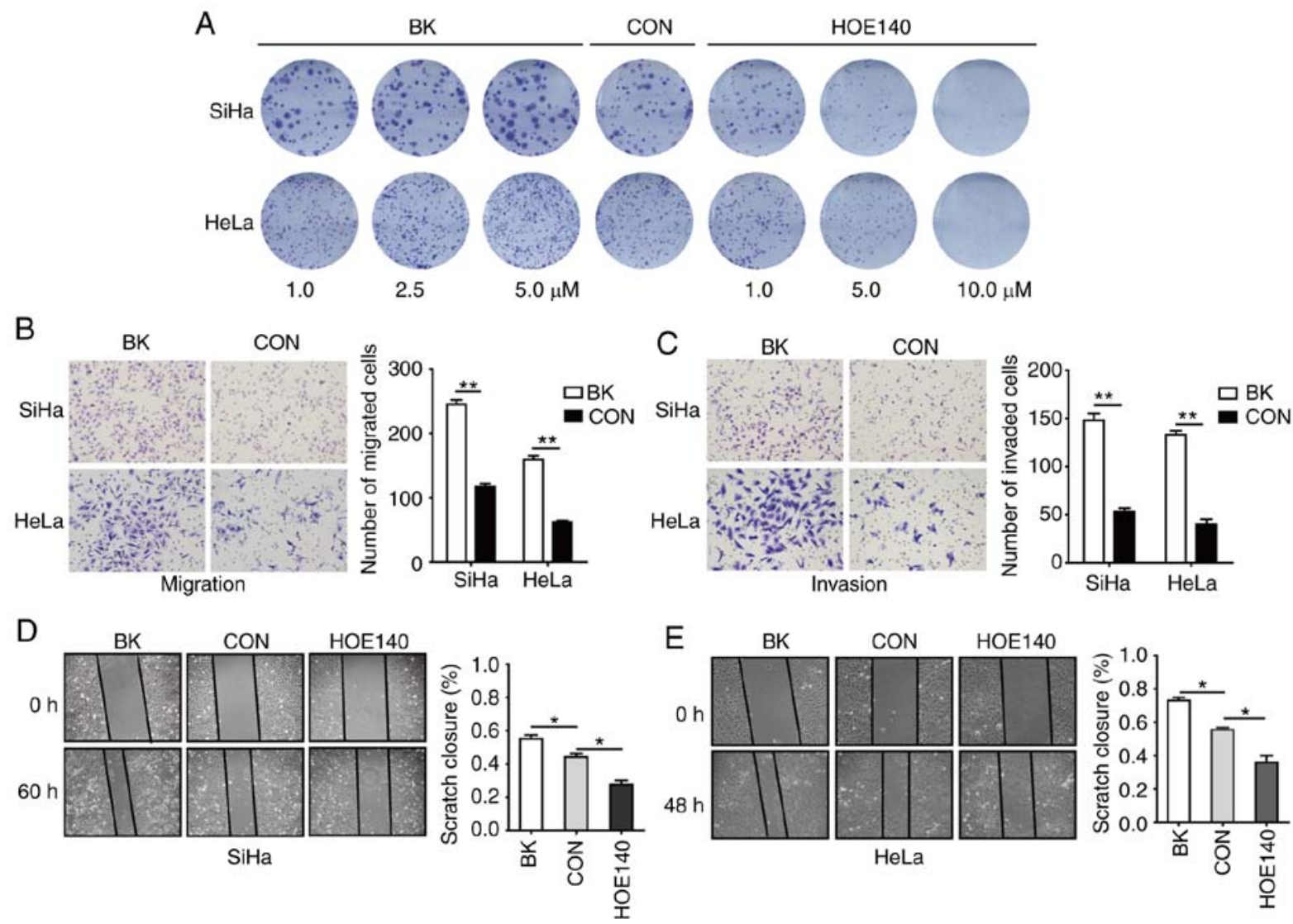

Figure 1. BK enhances the proliferation, migration, and invasion of CC cells. (A) Proliferation activity as detected by colony formation in SiHa and HeLa cells treated with different concentrations of BK or HOE140. (B and C) Transwell assays were used to assess cell migration and invasion in terms of cell numbers. (D and E) Representative images and quantification of the wound closure capacities of SiHa and HeLa cells. All experiments were performed in triplicate. Data are presented as the mean \pm SD. ${ }^{*} \mathrm{P}<0.05$ and ${ }^{* * *} \mathrm{P}<0.01$. BK, bradykinin; $\mathrm{CC}$, cervical cancer.

\section{Results}

BK enhances the proliferation, migration, and invasion of CC cells. To assess the potential role of BK in CC, colony formation, Transwell, and cell wound-healing assays were conducted. Plate colony formation assays were used to assess the proliferative activity of cells (14). In this assay, $\mathrm{SiHa}$ and HeLa cells were treated with $1,2.5$ or $5 \mu \mathrm{M}$ BK or 1,5 , or $10 \mu \mathrm{M}$ HOE140, respectively. As revealed in Fig. 1A, BK treatment resulted in a dose-dependent increase in cell proliferation, whereas HOE140 had the opposite effect, inhibiting cell proliferation in a concentration-dependent manner as compared with the control cells. Transwell assays revealed that BK enhanced the migration and invasion of human CC cells (Fig. 1B and C). In addition, cell wound-healing assays also revealed that $\mathrm{BK}$ promoted $\mathrm{CC}$ cell migration and that HOE140 blocked CC cell migration (Fig. 1D and E). These data indicated that $\mathrm{BK}$ promoted cell proliferation, migration, and invasion in CC cells.

Involvement of B2R in BK-mediated proliferation, migration, and invasion of $C C$ cells. To further determine whether $\mathrm{B} 2 \mathrm{R}$ is involved in the BK-mediated biological function of $\mathrm{CC}$ cell lines, B2R-overexpressed and -knockdown cell lines were constructed. After confirming mRNA and protein levels (Fig. 2A), plate cell colony formation assays were conducted. Fig. 2B revealed that $\mathrm{B} 2 \mathrm{R}$ overexpression significantly increased cell proliferation and that B2R knockdown markedly reduced cell proliferation. Transwell assays revealed that B2R-overexpressed CC cells exhibited greater migration and invasion abilities compared to those of the control cells, while in B2R-knockdown CC cells, the effect was reversed (Fig. 2C and D). The results of the scratch assays were similar to those of the Transwell assays (Fig. 2E and F). During the incubation period, $2.5 \mu \mathrm{M}$ BK was added to all cell supernatants. Collectively, these data indicated that BK promoted CC cell proliferation, migration, and invasion via $\mathrm{B} 2 \mathrm{R}$.

$B K$ upregulates MMP expression in CC cells. Whether MMPs are involved in BK-induced invasion and migration of CC cells was also investigated. Notably, treatment with BK resulted in a concentration-dependent upregulation of MMP2 and MMP9 in SiHa and HeLa cells (Fig. 3A and B). However, MMP2 and MMP9 expression was inhibited by HOE140 in a dose-dependent manner (Fig. 3A and 3B). Moreover, there was a positive association between $\mathrm{B} 2 \mathrm{R}$ expression and MMP2 and MMP9 expression (Fig. 4A and B). Thus, BK/B2R mediated migration and invasion of $\mathrm{CC}$ cells by increasing the expression of MMP2 and MMP9.

$B K$ enhances the activation of STAT3 signaling pathways to promote CC cell proliferation, migration, and invasion. Given that abnormal activation of STAT3 signaling plays an important role in CC cell proliferation, migration, and 

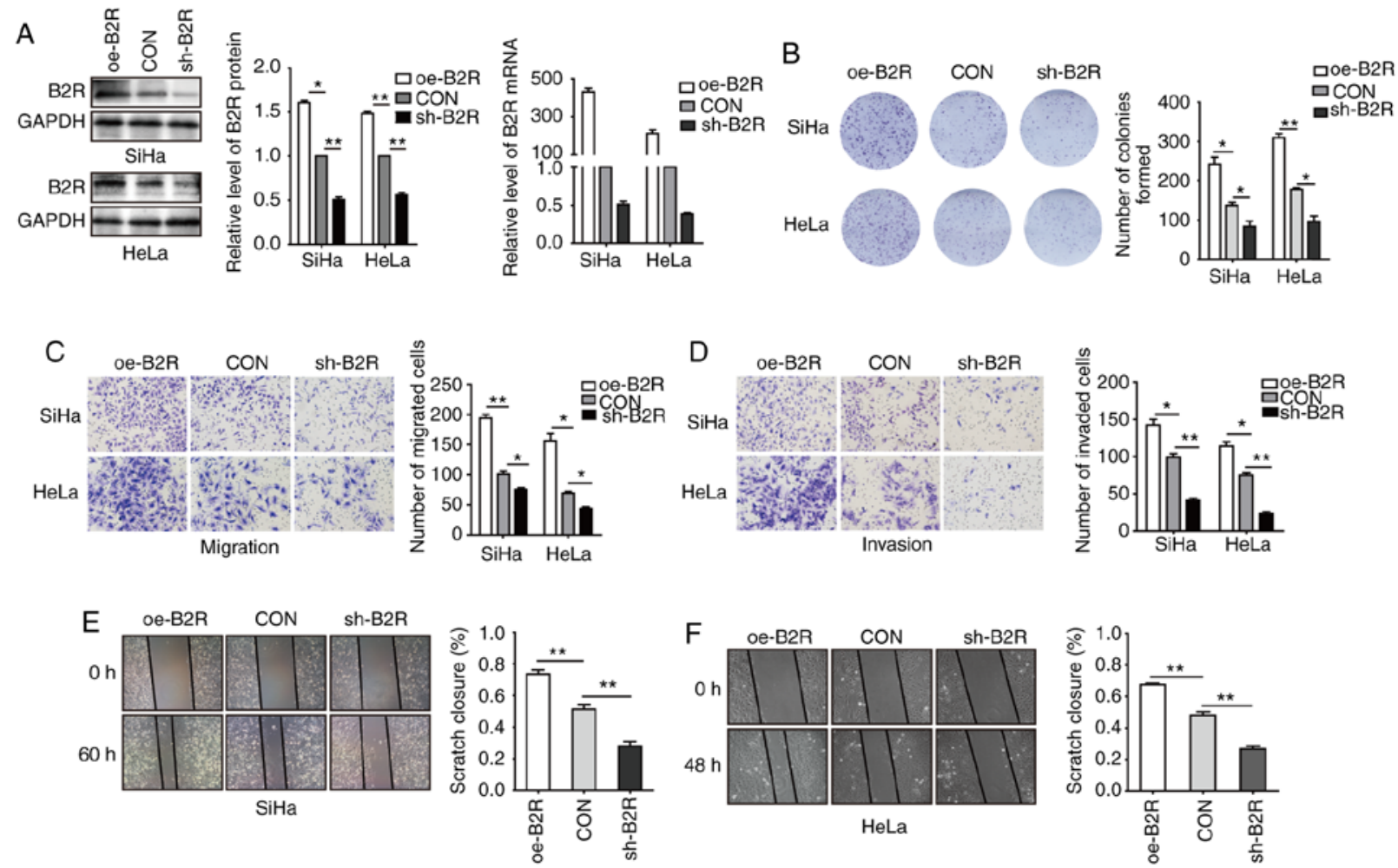

Figure 2. Overexpression or knockdown of B2R enhances or inhibits, respectively, the proliferation, migration, and invasion of CC cells. (A) SiHa and HeLa cells were transfected with oe-B2R, sh-B2R, or an empty lentiviral vector and analyzed with western blotting and RT-qPCR. (B) Plate colony formation assays were used to assess the proliferation activity of the established cells. (C and D) Transwell assays were used to assess cell migration and invasion by cell numbers. (E and F) Representative images and quantification of the wound closure capacities of the established cells. All experiments were performed in triplicate. Data are presented as the mean $\pm \mathrm{SD} .{ }^{*} \mathrm{P}<0.05$ and ${ }^{* *} \mathrm{P}<0.01$. B2R, bradykinin $\mathrm{B} 2$ receptor; CC, cervical cancer.

invasion $(23,24)$, whether STAT3 signaling pathways are involved in the BK-mediated biological function of CC cells was investigated. Western blotting indicated that phosphorylated (p)-STAT3 protein levels were increased after treatment with BK while HOE140 decreased p-STAT3 protein levels in a dose-dependent manner. For the STAT3-regulated pro-apoptotic protein cleaved caspase-9, the effect was reversed (Fig. 3A and B). Similarly, the protein level of p-STAT3 in B2R-overexpressed cells was markedly increased compared with that in the control cells, while B2R-knockdown $\mathrm{CC}$ cells exhibit the opposite effect. The expression of B2R also exhibited a positive association with those of MMP2 and MMP9 and a negative association with that of the apoptosis pathway-related protein cleaved caspase-9 (Fig. 4A and B). In summary, the present findings indicated that the activation of B2R and STAT3 played important roles during BK-induced proliferation, migration, and invasion in $\mathrm{CC}$ cells.

\section{Discussion}

$\mathrm{BK}$, the most prominent member of the kinin group, plays an important role in many cellular processes (25). An increasing number of studies have revealed that BK treatment promotes proliferation, angiogenesis, and metastasis in many cancers $(11,26)$. The present findings have revealed for the first time, to the best of our knowledge, that BK treatment promoted proliferation, migration, and invasion of CC cells, whereas the B2R inhibitor HOE140 exhibited the opposite effect. Previous research performed by our group determined that $\mathrm{BK}$ promoted $\mathrm{CC}$ angiogenesis via $\mathrm{B} 2 \mathrm{R}$ but not $\mathrm{B} 1 \mathrm{R}$ (12). Furthermore, in the present study, overexpression of B2R facilitated BK-treated CC cell proliferation, migration, and invasion, whereas downregulatoin of B2R blocked them. Collectively, the results of the present study indicated that $\mathrm{BK}$ plays a biological role via B2R in CC cells.

Several studies have demonstrated that BK promotes tumorigenesis through the MAPK, PKC, NF- $\kappa \mathrm{B}$, and ERK signaling pathways $(7,9,11,27)$. Constitutive STAT3 activation was revealed to be associated with various human cancers and indicated poor prognosis $(14,17,28,29)$. The present study revealed that treatment of $\mathrm{CC}$ cells with $\mathrm{BK}$ induced an increase in the expression of p-STAT3 Tyrosine705 (Y705) in a concentration-dependent manner. Moreover, the B2R antagonist HOE140 has the opposite effect. Activated STAT3 has been revealed to upregulate MMP expression and reduce the expression of pro-apoptotic proteins $(15,22)$. To the best of our knowledge, the present findings are the first to demonstrate that treatment of CC cells with BK/B2R upregulated p-STAT3 expression while it downregulated the expression of the pro-apoptotic protein cleaved caspase-9, consequently facilitating CC cell migration and invasion by stimulating the activity of MMP2 and MMP9, whereas silencing of B2R blocked these effects. These data confirmed that $\mathrm{BK} / \mathrm{B} 2 \mathrm{R}$ plays an important role in the malignant progression of $\mathrm{CC}$ by activating STAT3.

STAT3 plays an important role in tumorigenesis, and many studies have demonstrated that STAT3 inhibitors are promising agents for antitumor therapy (13). However, although small-molecule STAT3 inhibitors exhibit antitumor 

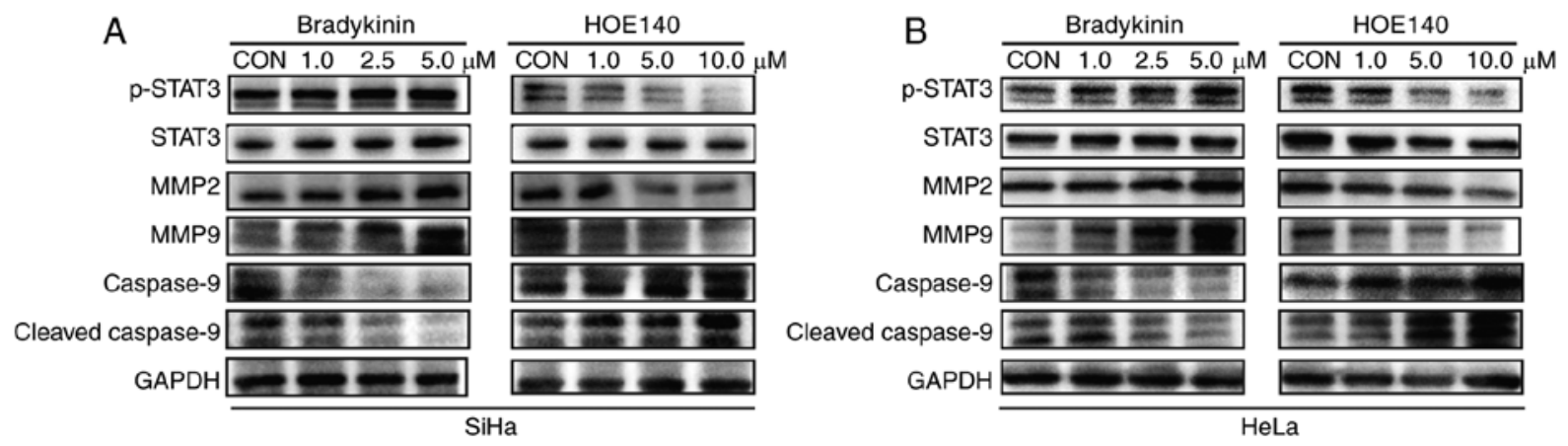

Figure 3. Representative blots revealing the protein levels of p-STAT3, STAT3, MMP2, MMP9, cleaved caspase-9, and caspase-9 in (A) SiHa and (B) HeLa cells treated with BK or HOE140 at different concentrations. GAPDH was loaded as a control. The results were similar for three independent experiments. p-STAT3, phosphorylated-STAT3; BK, bradykinin; MMP, matrix metalloprotease.
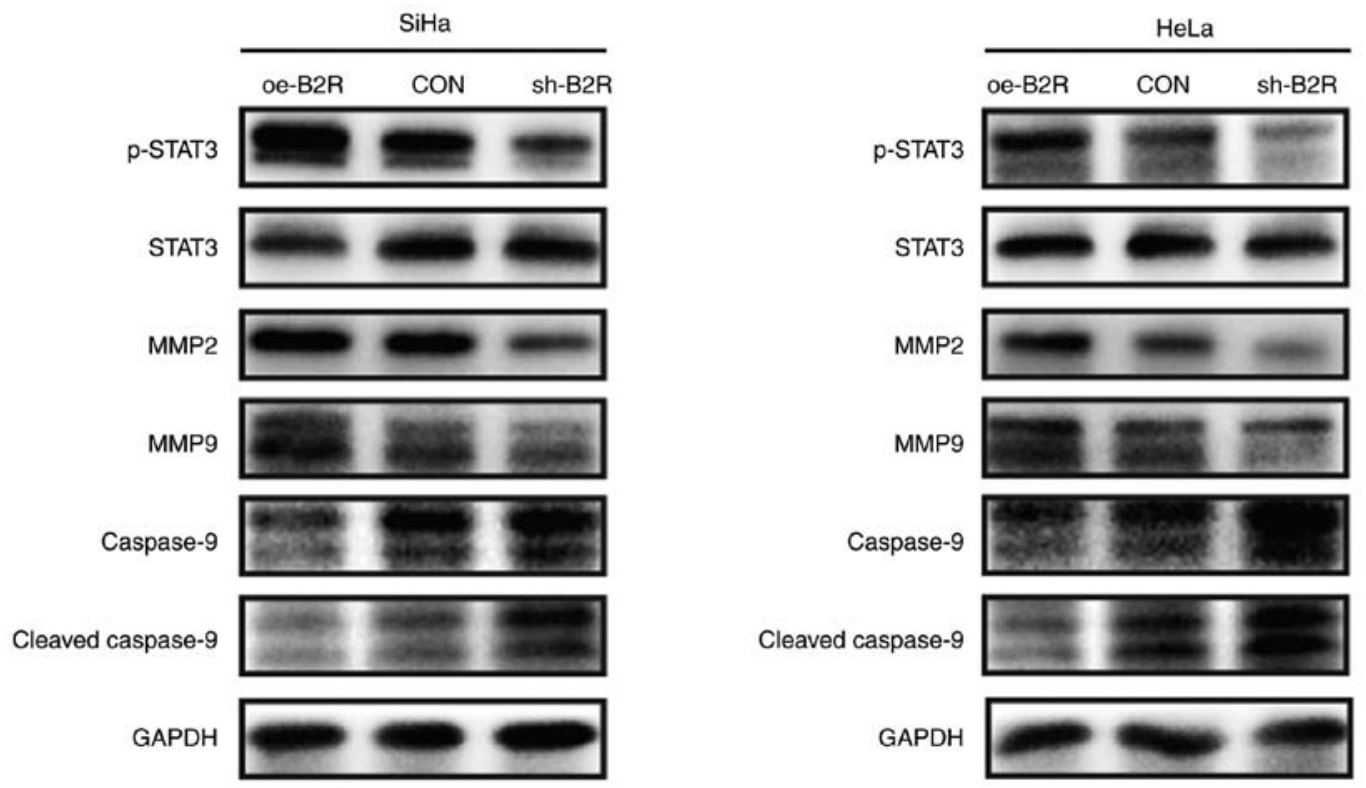

Figure 4. Representative blots revealing the protein levels of p-STAT3, STAT3, MMP2, MMP9, cleaved caspase-9, and caspase-9 in (A) SiHa and (B) HeLa cells transfected with oe-B2R, sh-B2R, or an empty lentiviral vector. GAPDH was loaded as a control. The results were similar for three independent experiments. p-STAT3, phosphorylated-STAT3; B2R, bradykinin B2 receptor; MMP, matrix metalloprotease.

effects, they also cause serious side effects, some of which are fatal $(30,31)$. The present study revealed that the B2R inhibitor HOE140 hindered the activation of STAT3, thus, it was speculated whether B2R inhibitors can also achieve the same antitumor effect as STAT3 inhibitors, but with reduced side effects. This is a potentially fruitful area of future research and may bring new hope to many cancer patients.

Notably, based on the biological function of BK in tumors, there are several studies that suggest that BK antagonists can be used as novel antitumor drugs (32). In the present study, the B2R antagonist HOE140 was revealed to inhibit the proliferation, invasion, and metastasis of $\mathrm{CC}$ cells. Moreover, Avdieiev et al reported that the combination of a BK receptor antagonist and thiazolidinone derivatives had a significant synergistic effect on fatality to rat and human glioma cells and also reduced drug resistance (33). BK antagonists have also been used in human trials and no harmful side effects have been observed. Thus, BK antagonists can be used for anticancer therapy (34). Accordingly, BK antagonists may serve as very promising agents for the treatment of $\mathrm{CC}$. For patients with recurrent $\mathrm{CC}$ and/or that are drug resistant, the combination of cisplatin/paclitaxel with Bevacizumab, an antibody directed against VEGF-A, provides a new treatment strategy which can increase the toxicity of drugs to tumor cells (35). For such patients, B2R antagonist treatment may also provide an option worthy of investigation.

In summary, it was demonstrated for the first time that BK promoted CC cell proliferation, migration, and invasion through activation of the B2 receptor and STAT3 signaling pathways. It was also revealed that BK/B2R facilitated CC cell migration and invasion by upregulating the expression of the STAT3-regulated products MMP2 and MMP9, and that BK/B2R downregulated the expression of the pro-apoptotic protein cleaved caspase- 9 . These findings will enhance our understanding of the mechanisms of CC tumorigenesis and metastasis, and they may provide an alternative to current $\mathrm{CC}$ therapies.

\section{Acknowledgements}

Not applicable. 


\section{Funding}

The present study was funded by the Natural Science Foundation Committee of China (NSFC81472444, NSFC81472783, NSFC81630060) and The Graduates' Innovation Fund of Huazhong University of Science and Technology (no. 2019ygscxcy060).

\section{Availability of data and materials}

All data used or analyzed during the current study are included in this published article.

\section{Authors' contributions}

WW conceived and designed the study, participated in every experiment, and drafted and critically revised the manuscript. YZ participated in experimental design and revised the manuscript. RW, GJ, FL, XC, XW analyzed the data. DM provided experimental technical support. LX provided final approval of the version to be published and was involved in the conception of the study. All authors revised, read and approved the final version of the manuscript and agree to be accountable for all aspects of the research in ensuring that the accuracy or integrity of any part of the work are appropriately investigated and resolved.

\section{Ethics approval and consent to participate}

Not applicable.

\section{Patient consent for publication}

Not applicable.

\section{Competing interests}

The authors declare that they have no competing interests.

\section{References}

1. Bray F, Ferlay J, Soerjomataram I, Siegel RL, Torre LA and Jemal A: Global cancer statistics 2018: GLOBOCAN estimates of incidence and mortality worldwide for 36 cancers in 185 countries. CA Cancer J Clin 68: 394-424, 2018.

2. Petignat $P$ and Roy M: Diagnosis and management of cervical cancer. BMJ 335: 765-768, 2007.

3. Moreau ME, Garbacki N, Molinaro G, Brown NJ, Marceau F and Adam A: The kallikrein-kinin system: Current and future pharmacological targets. J Pharmacol Sci 99: 6-38, 2005.

4. MaedaH,WuJ,OkamotoT,MaruoKand AkaikeT:Kallikrein-kinin in infection and cancer. Immunopharmacology 43: 115-128, 1999.

5. Searovic P, Alonso M, Oses C, Pereira-Flores K, Velarde V and Saez CG: Effect of tamoxifen and retinoic acid on bradykinin induced proliferation in MCF-7 cells. J Cell Biochem 106: 473-481, 2009

6. Wu J, Akaike T, Hayashida K, Okamoto T, Okuyama A and Maeda H: Enhanced vascular permeability in solid tumor involving peroxynitrite and matrix metalloproteinases. Jpn J Cancer Res 92: 439-451, 2001.

7. Yang WH, Chang JT, Hsu SF, Li TM, Cho DY, Huang CY, Fong $\mathrm{YC}$ and Tang $\mathrm{CH}$ : Bradykinin enhances cell migration in human chondrosarcoma cells through BK receptor signaling pathways. J Cell Biochem 109: 82-92, 2010.

8. Menke JG, Borkowski JA, Bierilo KK, MacNeil T, Derrick AW, Schneck KA, Ransom RW, Strader CD, Linemeyer DL and Hess JF: Expression cloning of a human B1 bradykinin receptor. J Biol Chem 269: 21583-21586, 1994.
9. Wang $\mathrm{G}$, Ye Y, Zhang $\mathrm{X}$ and Song J: Bradykinin stimulates IL-6 production and cell invasion in colorectal cancer cells. Oncol Rep 32: 1709-1714, 2014.

10. Vassou D, Notas G, Hatzoglou A, Castanas E and Kampa M: Opioids increase bladder cancer cell migration via bradykinin B2 receptors. Int J Oncol 39: 697-707, 2011.

11. Yu HS, Wang SW, Chang AC, Tai HC, Yeh HI, Lin YM and Tang CH: Bradykinin promotes vascular endothelial growth factor expression and increases angiogenesis in human prostate cancer cells. Biochem Pharmacol 87: 243-253, 2014.

12. Zhou Y, Wang W, Wei R, Jiang G, Li F, Chen X, Wang X, Long S, $\mathrm{Ma} \mathrm{D}$ and Xi L: Serum bradykinin levels as a diagnostic marker in cervical cancer with a potential mechanism to promote VEGF expression via BDKRB2. Int J Oncol 55: 131-141, 2019.

13. Wang X, Crowe PJ, Goldstein D and Yang JL: STAT3 inhibition, a novel approach to enhancing targeted therapy in human cancers (Review). Int J Oncol 41: 1181-1191, 2012.

14. Yao S, Xu J, Zhao K, Song P, Yan Q, Fan W, Li W and Lu C: Down-regulation of HPGD by miR-146b-3p promotes cervical cancer cell proliferation, migration and anchorage-independent growth through activation of STAT3 and AKT pathways. Cell Death Dis 9: 1055, 2018.

15. Saini U, Naidu S, ElNaggar AC, Bid HK, Wallbillich JJ, Bixel K, Bolyard C, Suarez AA, Kaur B, Kuppusamy, et al: Elevated STAT3 expression in ovarian cancer ascites promotes invasion and metastasis: A potential therapeutic target. Oncogene 36: 168-181, 2017.

16. Alvarez JV, Greulich H, Sellers WR, Meyerson M and Frank DA: Signal transducer and activator of transcription 3 is required for the oncogenic effects of non-small-cell lung cancer-associated mutations of the epidermal growth factor receptor. Cancer Res 66: 3162-3168, 2006.

17. Yang S, Yang C, Yu F, Ding W, Hu Y, Cheng F, Zhang F, Guan B, Wang $X$ and Lu L and Rao J: Endoplasmic reticulum resident oxidase ERO1-Lalpha promotes hepatocellular carcinoma metastasis and angiogenesis through the S1PR1/STAT3/VEGF-A pathway. Cell Death Dis 9: 1105, 2018.

18. Pradeep S, Huang J, Mora EM, Nick AM, Cho MS, Wu SY, Noh K, Pecot CV, Rupaimoole R, Stein MA, et al: Erythropoietin stimulates tumor growth via EphB4. Cancer Cell 28: 610-622, 2015.

19. Smith HA and Kang Y: The metastasis-promoting roles of tumor-associated immune cells. J Mol Med (Berl) 91: 411-429, 2013.

20. Liu Z, Tian Z, Cao K, Zhang B, Wen Q, Zhou X, Yang W, Wang T, Shi H and Wang R: TSG101 promotes the proliferation, migration and invasion of hepatocellular carcinoma cells by regulating the PEG10. J Cell Mol Med 23: 70-82, 2019.

21. Shishodia G, Shukla S, Srivastava Y, Masaldan S, Mehta S, Bhambhani S, Sharma S, Mehrotra R, Das BC and Bharti AC: Alterations in microRNAs miR-21 and let-7a correlate with aberrant STAT3 signaling and downstream effects during cervical carcinogenesis. Mol Cancer 14: 116, 2015.

22. Guha P, Gardell J, Darpolor J, Cunetta M, Lima M, Miller G, Espat NJ, Junghans RP and Katz SC: STAT3 inhibition induces Bax-dependent apoptosis in liver tumor myeloid-derived suppressor cells. Oncogene 38: 533-548, 2019.

23. Zhang J, Jia J, Zhao L, Li X, Xie Q, Chen X, Wang J and Lu F: Down-regulation of microRNA-9 leads to activation of IL-6/Jak/STAT3 pathway through directly targeting IL-6 in HeLa cell. Mol Carcinog 55: 732-742, 2016.

24. Zhao H, Wang W, Zhao Q, Hu G, Deng K and Liu Y: BCL3 exerts an oncogenic function by regulating STAT3 in human cervical cancer. Onco Targets Ther 9: 6619-6629, 2016.

25. da Costa PL, Sirois P, Tannock IF and Chammas R: The role of kinin receptors in cancer and therapeutic opportunities. Cancer Lett 345: 27-38, 2014.

26. Wang G, Sun J, Liu G, Fu Y and Zhang X: Bradykinin promotes cell proliferation, migration, invasion, and tumor growth of gastric cancer through ERK signaling pathway. J Cell Biochem 118: 4444-4453, 2017.

27. Sabatini F, Luppi F, Petecchia L, Stefano AD, Longo AM, Eva A, Vanni C, Hiemstra PS, Sterk PJ, Sorbello V, et al: Bradykinin-induced asthmatic fibroblast/myofibroblast activities via bradykinin B2 receptor and different MAPK pathways. Eur J Pharmacol 710: 100-109, 2013.

28. Morikawa T, Baba Y, Yamauchi M, Kuchiba A, Nosho K, Shima K, Tanaka N, Huttenhower C, Frank DA, Fuchs CS and Ogino S: STAT3 expression, molecular features, inflammation patterns, and prognosis in a database of 724 colorectal cancers. Clin Cancer Res 17: 1452-1462, 2011. 
29. Yin W, Cheepala S, Roberts JN, Syson-Chan K, DiGiovanni J and Clifford JL: Active Stat3 is required for survival of human squamous cell carcinoma cells in serum-free conditions. Mol Cancer 5: 15, 2006.

30. Wong AL, Soo RA, Tan DS, Lee SC, Lim JS, Marban PC, Kong LR, Lee YJ, Wang LZ, Thuya WL, et al: Phase I and biomarker study of OPB-51602, a novel signal transducer and activator of transcription (STAT) 3 inhibitor, in patients with refractory solid malignancies. Ann Oncol 26: 998-1005, 2015.

31. Murakami T, Takigawa N, Ninomiya T, Ochi N, Yasugi M, Honda Y, Kubo T, Ichihara E, Hotta K, Tanimoto M and Kiura K Effect of AZD1480 in an epidermal growth factor receptor-driven lung cancer model. Lung Cancer 83: 30-36, 2014.

32. Stewart JM: Bradykinin antagonists as anti-cancer agents. Curr Pharm Des 9: 2036-2042, 2003.
33. Avdieiev S, Gera L, Havrylyuk D, Hodges RS, Lesyk R, Ribrag V, Vassetzky Y and Kavsan V: Bradykinin antagonists and thiazolidinone derivatives as new potential anti-cancer compounds. Bioorg Med Chem 22: 3815-3823, 2014.

34. Stewart JM, Gera L, Chan DC, Bunn PJ Jr, York EJ, Simkeviciene V and Helfrich B: Bradykinin-related compounds as new drugs for cancer and inflammation. Can J Physiol Pharmacol 80: 275-280, 2002.

35. Monk BJ, Sill MW, Burger RA, Gray HJ, Buekers TE and Roman LD: Phase II trial of bevacizumab in the treatment of persistent or recurrent squamous cell carcinoma of the cervix: A gynecologic oncology group study. J Clin Oncol 27: 1069-1074, 2009.

(i) (9) This work is licensed under a Creative Commons

EY NO ND Attribution-NonCommercial-NoDerivatives 4.0 International (CC BY-NC-ND 4.0) License. 